Images vol. XXIX/no. 38

Poznań 2021

ISSN 1731-45OX

\title{
The experience and exploration of worlds in single-player video games
}

\begin{abstract}
Aвstract. Wolf Mark J.P., The experience and exploration of worlds in single-player video games. "Images" vol. XXIX, no. 38. Poznań 2021. Adam Mickiewicz University Press. Pp. 223-230. ISSN 1731-450X. DOI 10.14746/i.2021.38.14.

The author indicates a few of the most common experiences that are encountered, particularly in single-player open-world games where exploration of the world plays a large part of the enjoyment of a game. The experiences mentioned in the essay are: arrival in the world, the gaining of an objective, the revelation of the expansiveness of the world, the first contact with the world's residents, world routines, travel to the world's boundaries, and the completist's exhaustion of the world when one has seen and experienced everything.
\end{abstract}

KEYWORDS: game world, open world, single-player video games, exploration, narrative

Today more than ever, a large part of the pleasure of playing video games is the vicarious visiting of an imaginary world. In many exploratory games and open-world games, from Zork (1977), Myst (1993), and Riven (1997), to The Unfinished Swan (2012), The Vanishing of Ethan Carter (2014), and Obduction (2016), exploration is the only real objective the player has at the start of a game; one is dropped into the world with little or no instructions or explanation, left to wander the world. [1] It is up to the player to learn the geography and mechanics of the world, two areas which offer great opportunities to integrate puzzles into a world. A classic example of this occurs right at the beginning of Myst; the player arrives on the dock and advances forward along it to an unmarked switch. Even though there is no indication as to what the switch might do, the player moves the switch anyway; it is something to do, after all. After the switch is thrown, nothing at all appears to happen, the clever first twist in the game. The simplest expectation that we could have, that something will happen, is thwarted. We expect to learn something of the world's mechanics from moving the switch back and forth, but we learn nothing, except a hint as to how puzzling the visit to this world will be.[2]

[1] Technically speaking, there may be a vague or general kind of objective given; at the start of The Vanishing of Ethan Carter, we are told to find Ethan; in Fallout 4, we are supposed to find Shaun; and in Riven, we are asked to capture Gehn and find Catherine; but in all these cases, we will have to explore extensively before we can do anything even remotely connected to finding these people. So, in a sense, such objectives really do not orient us much more than if we did not have them.

[2] Myst's sequel, Riven, thwarts us even more at the beginning; as soon as we arrive on Riven, looking out into the world, bars ascend, trapping us in our starting position before we have even had a chance to step forward, and the book Atrus has given the player is stolen right away by a guard. 
While it has now become rare for a game to include a manual explaining the game's world, we often have other hints: the on-line art (or box or packaging art, which still survives for some games), game trailers and other promotional material, and even genre conventions and game conventions that tell us what to expect and how to use the game controller; controller instructions are also available as an option within some games. Yet when it comes to world exploration, the best game trailers will only tease us with brief glimpses of what is to come, revealing the graphical and aesthetic style of the game, whether photorealistic or stylized, steampunk or pastoral, urban or rural, fantastic or quotidian, period or present day, and these might even be calculated to mislead, or to withhold information. Likewise, conventions can be overturned or played with, as Myst's opening shows. At any rate, the first phase of the experience and exploration of a world is that of arrival, or our starting point, which includes the direction we are facing when the first interactive imagery appears. In a three-dimensional world, there is a tendency to move forward, and the player is often encouraged to do so, as there is usually something beckoning further in along the $\mathrm{z}$-axis, a short distance away. The cautious player will turn in all directions first, to gain his or her bearings, but quite often, we are only on the edge of the world, and forward is the only way to go. For example, in our initial position on Myst Island's dock, there is a rock wall behind us and to the left, and only the ocean and sunken ship to the right, so we must go forward. The Vanishing of Ethan Carter provides an interesting twist to this kind of beginning; we emerge from a tunnel, looking down a train track in a wooded area. While we can, of course, follow the track into the distance, we can also turn around and go back into the tunnel, to see where it goes; after a long walk through it, we find the other end of the tunnel emerges at the same place as that at where we appeared upon arriving (though we are given a Tunnel Achievement trophy for our trouble).

Our arrival is often made to appear irreversible: we click on the Myst book and enter Myst Island, with no way back; we travel through the storm in a rowboat to the lighthouse in Bioshock Infinite (2013), and from there our trip to the flying city of Columbia is one-way; in $\mathrm{Ob}$ duction, the seed pod transports us to the entry canyon and we cannot return. Since we have come from somewhere, one objective could be simply to get back to where we came from, and the irreversibility of our arrival means we have to find a different way to get back. Naturally, it is usually only at the very end of the game that we are able to leave (apart from dying there, if that is even possible). In some cases, like Fallout 4 (2015), events have changed the world (in this case, nuclear war) and there is nowhere to go, so another objective becomes necessary.

The arrival sequence may also include an entrance area of sorts (which sometimes cannot even be returned to), in which we learn the basic mechanics of interaction in the world, receive objects we may need, or even interact with a non-player character. Finally, when we 
leave this entrance area, we step out into the world, and are given a sense of its expansiveness, through vistas that open before us, revealing faraway objects and places on the horizons; glimpses of the depths of the world; or long distances through which we walk or ride to get to our first salient location where story information is waiting for us. The story information, often in the form of messages from non-player characters, may give us an indication of what to do, but the very expansiveness of the world itself gives us goals; locations visible in the distance are, in one sense, implied exploration goals, especially if they are blocked by locked doors or gates, impassable terrain, or other obstacles, and we usually are given some sense of what areas will be accessible as opposed to those which simply function as a visual backdrop at the edge of the world. And, expansive as the world may seem, at some later point, we usually will have an experience in which we encounter another, previously unseen expanse, either extending from the first expanse seen, or of an entirely new area (often encountered after travel through a portal); after two such expanses are experienced, there is the feeling that still others could occur, and the surprise created by the second experience makes us wonder just how large the world is, and whether more spaces remain to be revealed (one of the best exploration experiences is to be amazed at the size of a world, only to find later that you have underestimated its size). Or, we may encounter a variety of places along with a sense of gradually approaching some distant destination; in Journey (2012), we see the mountain with the beam of light in the distance, and gradually get closer and closer to it, until we finally ascend the mountain to get to the valley from which the beam of light originates.

Another moment in world exploration is the first contact with one of the game's non-player characters. Many open game worlds, like many of those mentioned above, are vast, empty places devoid of characters; the lonely landscape is often narratively explained through some tragic past event, such as postapocalyptic worlds whose citizens died in a war or some other calamity, or worlds where everyone has left for somewhere else, gone into hiding, or been taken somewhere against their will. These empty landscapes can make the player more forgiving of the limitations of artificial intelligence when a character finally is encountered. Improvements in AI are making more interaction possible, but currently, the best solutions to the problem of interactivity involve narratively limited interaction. Non-player characters are either not interested in interacting (like the citizens walking or standing around various locations in Bioshock Infinite or Marvel's Spider-Man (2018)), or they shoot or attack the player's avatar before any conversation can occur, as in so many action-based shooting games. Some characters are scripted to advance the story, with limited interaction provided (as in Heavy Rain (2010) and Fallout 4, where the player is given four or five responses to choose from when conversing with the game's characters). And, of course, there are the sidekicks who travel with the main character, exchanging quips and aiding in fights, like Elizabeth in Bioshock 
Infinite and Ellie in The Last of Us (2013). As AI improves, so does the illusion of companionship provided by such characters, though their algorithmic (or even scripted) nature still remains apparent in most cases (in both Bioshock Infinite and The Last of Us, the player merely observes the sidekick and the named main character having a conversation and does not choose what is said). Quite often, though, in more open-world games, there is relatively little contact with non-player characters, due to their scarcity.

Worlds with more linear narratives, which are often very mission-based, are typically more populous and thriving, like those of the Grand Theft Auto and Red Dead Redemption franchises. But the most open and explorable worlds are more likely to be the kind of empty ones described above, which often exist in some post-disaster state. While there are a number of tropes associated with such worlds, let us examine in detail one that is encountered in many worlds; the presence of ruins (sometimes an entire world is in ruins), which serve a number of purposes that aid world design while also heightening the player's experience. First, ruins give a location a "before and after" dual timeframe; a sad melancholy present, and a supposedly better past when everything was new and functional. Looking through ruins, we often can guess at past events that happened, due to clues that remain: walls and floors that collapsed, places that were broken into or looted, the detritus of armed conflict or struggles, rooms and vehicles that fell into disrepair, or crumbling buildings and broken stretches of concrete now overgrown and reclaimed by nature. Rust, dust, dirt, cobwebs, patinas, clutter, torn fabric gently swaying in the breeze; every object and surface shows signs of wear and disuse, giving places more than just a realistic "lived-in" look and more of what we might call a "lived-in and died-in" look. Instead of computer-generated assets that look phony because of their flawlessness, even surfaces, and perfection, ruins give everything finely-detailed wear-and-tear, which is referred to in Japanese aesthetics as wabi-sabi. Such damage may only be represented by a texture map applied to a simple object, but it still enhances the realism of the graphics. And if everything is carefully designed, one can piece together past events, giving the world narrative depth as the player imagines both what the world used to look like and what happened to it.

Second, ruins can be used to shape the player's experience within a location. The ruined state of a place justifies why relatively few things are usable or worth picking up for a player's inventory; the few good items are hidden amongst piles of junk and wreckage, making the things you can find and use seem even more valuable by contrast. The ruined state of locations also explains why the inhabitants are gone, or why buildings can be entered that would normally be secured against trespassers. Things like collapsed ceilings, fallen timbers, burned-out interiors, and so on, can also be strategically arranged so as to naturally block doorways or passageways, forcing players to go around obstacles, and subtly limit or direct player movement without appearing to do 
so. Things like bridges or walkways can be dilapidated and uncrossable, suggesting areas that the player either cannot visit or that require other means of access. Some damage, like holes in the floor, can offer glimpses, or even one-way passage, into other areas; the player can jump down or slide down, but not get back up again. In dynamic locations, structures can suddenly collapse and force the player in a new direction, cutting off existing routes already traveled. Many game puzzles involve working with ruined locations, as the player must find ways to get the power turned on again, assemble or repair broken machinery, and start up machinery that appears to have lain dormant a long time; such actions can be more rewarding than simply turning on new and functioning machinery would be.

Third, ruins often convey a heavy sense of mood and atmosphere. There is usually a sad and melancholy feel to locations laying in ruin; interiors are often in shadow with harsh sunlight filtering through into rooms, as a place's ambience even seems more ominous when it comes in the wake of destruction. Ruins can help generate a variety of emotional responses; on the one hand, there is the sad, heavy, gothic feel of dark, ruined interiors rife with shadows and dark corners, verging on fear and anxiety if combined with the expectation of sudden violence from hidden adversaries. On the other hand, ruins can be experienced outdoors in late afternoon sunlight, giving them a more melancholy and contemplative feel, one which may be softened by the natural surroundings and ambience they are set in, where trees, plants, rocks, and flowing rivers and the effects of wind provide a feeling of life to counter the deadness and silent stillness of the ruins. Ruins may also indicate lost cultures or civilizations, and the design of the ruins can suggest who those people were and what they valued, or did not value (for example, clues indicating refined intelligence like writings versus clues indicating brutal rituals of human sacrifice). Ruins have become a popular trope in open-world games for their aesthetic value and strategic usefulness. Finally, ruins can simplify a location by limiting what the player can do there, sometimes keeping activity to few basic routines.

Just as the player becomes accustomed to world geography, there are the world routines that are performed over and over, some with variations, some not. Many routines involve collecting, for example, weapons, ammunition, fuel, food and drink, medical supplies, items for crafting, expository materials (paper messages, recordings, photographs, and so on), and information (door codes, passwords, lock combinations, etc.). Other routines involve the use of the things collected, such as loading and using weapons, eating food, healing damage, assembling and crafting items, battling enemies, and so forth. We can divide world routines into two categories: ones in which the player-character gains something positive, and ones in which the player-character avoids something negative. The first type of routine moves the player forward in a positive direction, such as when a player-character is leveling up into 
a series of more powerful positions or is gaining abilities (or increasing the numbers that represent ability levels); the player-character gains something from the repeated actions. The second type of routine is repeated simply to allow the player-character to continue surviving, such as eating, drinking, and the healing of wounds or damage, or keeping up an inventory of needed items (like ammunition, when among foes, or air, when underwater); in these cases, instead of gaining something, the player-character is merely keeping from losing something (usually, the player-character's life). Games will often feature both types of routines, and may even force the player to choose between the two types, for example, when currency can buy either food and medical supplies or weapons and ammunition; or when a player is simply limited by how much can be carried at any given time. The more complex worlds become, the more they will have in common with resource management games, along with all the objectives and skill requirements found in such games.

Once world routines become second nature, they shape the player's experience of the world, as the player plans encounters with non-player characters and locations based on the finding and usage of these resources; at worst, this entails "grinding", the repetitive and tedious completion of tasks in order to gain points, reduce damage, or fend off foes, actions which can become a negative experience if they are required too often or without enough variation. (Sometimes the premise behind actions can even become ridiculous; for example, in Bioshock Infinite, why does one's health improve after you eat cotton candy found in a garbage can?) Yet amidst all the repetitive routines required by gameplay, the story (or the open-world experience, if there is no predetermined story) must keep advancing if the game is to remain interesting, and this is often done through the continued revelation of world details which construct backstory, link narrative threads, and further enrich our understanding - and thus our experience - of the world.

Finally, there comes a point in the player's experience of a game's world where the boundaries of the game's space become evident. However cleverly they are camouflaged, they cannot be traversed, and so they signal the finite size of the world, no matter how big it may be.[3] Boundaries can be naturally motivated by the game's terrain, appearing as the shores of an ocean, impassable mountains, uncrossable rivers, cliff edges and abysses, darkness, or even the curvature of a planet's surface that brings the player back to his or her starting point. Some-

[3] True, in some cases like Elite: Dangerous (2014) and No Man's Sky (2016), the worlds are made up of billions of planets, most of which players will never see; while these games' world are well beyond what any player can fully explore, the algorithmic nature of their procedurally generated content may start to feel like theme and variation, the more one experiences of it, until it, too, begins to seem repetitive (the worlds of Minecraft (2011) are a good an example of this). Perhaps only games with large numbers of other human players, like EVE On-line (2003), are able to have continually evolving worlds which can hold a player's interest for long periods of time. 
times boundaries are little more than invisible forces; in the relatively small world of Flower (2009), the player who keeps trying to move in one direction is eventually redirected and turned back toward the game's spaces. In other games, like Red Dead Redemption (2010) or Marvel's Spider-Man (2018), going too far afield from one's mission can bring about a warning that has the player turn back. In other cases, going too far in one direction merely leads to the player-character's death.[4] In most cases, the world's geographical borders also feature visual backdrops that provide vistas of what is in the distance beyond the edge of the navigable game world, even though one cannot go there, similar to the painted backdrops that are integrated into dioramas. The world's edges limit exploration, and are often used to keep players on a somewhat linear narrative track in which geographic progression is closely tied to narrative progression. Eventually, a player will have explored the world's geography exhaustively, and all that will remain will be the traversing of all the narrative threads to their ends. Although a game cannot ensure that geographic exploration will not be exhausted before the narrative exploration is (even in an open world where exploration is allowed), the main narrative threads can keep from using all of the geographic areas of a world, saving locations purely for side missions or even Easter eggs, which are only found with difficulty, or even not using them at all.

Eventually, the completist player will have explored all of the game world's geography and seen all of the narrative lines and outcomes in the game's story (when present). While many games have end sequences that give closure to their narratives, some will actually allow the player to remain in the world afterward (such as in Myst, The Vanishing of Ethan Carter, and Obduction), to further wander around if the player desires, even though there is nothing more to do. This is, indeed, one of the strangest experiences that one can have in a game world; with the sudden absence of narrative drive, enemies to fight, or enigmas to be unraveled, there is a feeling of emptiness, even loneliness, in a world in which there is nothing more to see and in which nothing more will happen. The game objectives have been met, yet the player is not forced to leave. Even if there are a few additional trophies or Easter eggs for the player to find, remaining in the world can seem rather anticlimactic.

On the other hand, the completist's exhaustion of a game world, whether one can stay in it or not, can be a satisfying experience, like the finishing of a long novel or television series; especially if there was a complete narrative structure, and particularly one with a point to it, as one would expect to find in literature, film, or other narrative media. Yet, even without a narrative, a world can feel complete and purposeful if it has a logic and structure to it, especially if there are ongoing systems

[4] Going all the way north in Red Dead Redemption 2 (2018) can result in falling off the map; see the video "Reach North Pole (Out of Map) in Red Dead
Redemption 2 (RDR2) - Beyond Tempest Rim (Canada)", December 24, 2018, at <https://www.youtube. com/watch? $\mathrm{v}=\mathrm{hKBoXr9RPlQ}>$. 
generating events, as in the world of Minecraft, with its simple creatures and animals moving about, or the worlds of multiplayer games where other players inhabit the world in an ongoing basis. Such worlds invite the player to return, and even may hold the promise of changes that will occur during the player's absence, although players looking for persistent worlds will more likely find them in on-line multiplayer games (here, too, one can find overlap; even though Journey (2012) is essentially a single-player game, one can encounter other player-characters wandering the world, and their user names are revealed at the end of the game).

The worlds of video games have become so detailed and varied that it is impossible to cover all of the kinds of experiences that one can have in them within a single essay; I have tried here to indicate a few of the most common experiences that are encountered, particularly in single-player open-world games where exploration of the world plays a large part of the enjoyment of a game. All of the experiences mentioned above- arrival in the world, the gaining of an objective, the revelation of the expansiveness of the world, the first contact with the world's residents, world routines, travel to the world's boundaries, and the completist's exhaustion of the world when one has seen and experienced everything - are things that need to be examined and discussed further, along with the tropes that have come to be associated with them (such as ruins, described above). While much has been written regarding the experience of narrative material in games, these other experiences, which may or may not occur as part of a predetermined narrative, are individually worth investigating, particularly as the experience of the worlds of video games are so different from that of worlds seen through other forms of media. 